\title{
1 Computational optical sectioning by phase-space imaging with 2 an incoherent multiscale scattering model
}

3 Yi Zhang ${ }^{1,2^{*}}$, Zhi $\mathrm{Lu}^{1,2^{*}}$, Jiamin $\mathrm{Wu}^{1,2^{*}, \dagger}$, Xing $\operatorname{Lin}^{1,2,3}$, Dong Jiang ${ }^{4}$, Yeyi Cai ${ }^{1}$, Jiachen

$4 \mathrm{Xie}^{1,2}$, Tianyi Zhu ${ }^{1,2}$, Xiangyang $\mathrm{Ji}^{1,2 \dagger}$ \& Qionghai Dai ${ }^{1,2,3 \dagger}$

$5 \quad{ }^{1}$ Department of Automation, Tsinghua University, Beijing, 100084, China

$6 \quad{ }^{2}$ Institute for Brain and Cognitive Sciences, Tsinghua University, Beijing 100084, China

$7 \quad{ }^{3}$ Beijing Innovation Centre for Future Chips, Tsinghua University, Beijing 100084, China

$8{ }^{4}$ State Key Laboratory of Membrane Biology, Tsinghua University-Peking University

9 Joint Centre for Life Sciences, Beijing Frontier Research Centre for Biological Structure,

10 School of Life Sciences, Tsinghua University, Beijing, 100084, China

$12{ }^{*}$ These authors contributed equally to this work

14 †Correspondence: wujiamin@tsinghua.edu.cn (J.W.), xyji@tsinghua.edu.cn (X.J.),

15 qhdai@tsinghua.edu.cn (Q.D.) 
17 Optical sectioning is essential for fluorescence imaging in thick tissue to extract infocus information from noisy background. Traditional methods achieve optical sectioning by rejecting the out-of-focus photons at a cost of photon efficiency, resulting in a tradeoff between sectioning capability and detection parallelization. Here, we show phase-space imaging with an incoherent multiscale scattering model can achieve computational optical sectioning with $\sim 20 \mathrm{~dB}$ improvement for signalto-background ratio in scattering medium, while maximizing the detection parallelization by imaging the entire volume simultaneously. We validated the superior performance by imaging various biological dynamics in Drosophila embryos, zebrafish larvae, and mice.

The beauty of life lies in the complexity and variety of cellular behaviors in threedimensional (3D) living organisms, which are difficult to appreciate without optical sectioning $^{1-3}$, as the information can be easily flooded in the severe noisy background.

30 Various methods have been proposed to achieve optical sectioning by rejecting the outof-focus photons either physically or computationally, such as confocal microscopy ${ }^{4,5}$, two-photon microscopy ${ }^{6,7}$, light-sheet microscopy 8,9 , and structured illumination microscopy ${ }^{10,11}$. However, these methods still require scanning of points, lines, or planes for 3D imaging, leading to the tradeoff between the sectioning capability and detection parallelization $^{1,3,12}$. Such a tradeoff intrinsically restricts the 3D imaging speed in fluorescence imaging, especially with a limited photon budget set by the sample health ${ }^{1,12,13}$. In addition, it's hard to remove the scattered photons due to the lack of depthdependent features.

Phase-space imaging, by collecting the high-dimensional local variances of the coherence, provides a new imaging framework with digital synthesis of the partially-

41 coherent light field, which shows strong robustness to scattering and aberrations ${ }^{14-18}$. As 42 a typical example, light-field microscopy $(\text { LFM })^{19}$ captures the phase-space 
43 measurements within a snapshot by a microlens array ${ }^{20}$, facilitating various applications 44 in biology, such as imaging hemodynamics ${ }^{21}$ and large-scale neural activities ${ }^{14,22}$. 45 Different from wide-field imaging with a shallow depth of field (DOF) ${ }^{23-25}$, LFM keeps 46 the photons focused along different angles within the extended depth of field and maximize the parallelization by imaging the entire volume simultaneously. However, it suffers from great degradation in thick tissue such as the mammalian brain, due to scattering and dense fluorescence labeling. While many efforts in hardware have been made to achieve additional optical sectioning ${ }^{26-28}$ at the cost of temporal resolution, space constraints and system compactness, the imaging model of this computational framework has barely been explored, especially for complicated imaging environment in deep tissue $e^{29,30}$.

Here, we show the necessity of an accurate imaging model in the complete space to unlock the full power of phase-space imaging in thick tissue. With an incoherent multiscale multiple-scattering model, phase-space imaging can achieve snapshot quantitative $3 \mathrm{D}$ information with optical sectioning computationally in densely labeled or scattering samples. This method is termed as quantitative LFM (QLFM). We find that the severe degradation of traditional LFM in thick tissue mainly results from the incomplete space and ideal imaging model utilized during reconstruction ${ }^{14,29,30}$. By

61 building up the incoherent imaging process in phase-space domain accurately with various factors, including the nonuniform resolution of different axial planes, out-offocus fluorescence across a large range, multiple scattering, and system aberrations, we can not only improve the resolution and contrast with significantly-reduced computational costs, but also achieve two-orders-of-magnitude improvement in signalto-background ratio (SBR) over traditional algorithms and wide-field microscopy (WFM), which is critical for quantitative biological analysis. To demonstrate the versatile applications, we imaged various 3D biological dynamics in different specimens, including the zebrafish larvae heart beating, blood flow, and whole-brain neural activity, 
70 Drosophila embryo development, and mouse brain neural activity with notably better

71 performance than that of traditional ideal models without increasing the system 72 complexity. 


\section{Results}

\section{$74 \quad$ Principle and implementation of QLFM}

As a general computational model for incoherent conditions, our QLFM method is compatible with different schematics of phase-space imaging. Here, we chose the unfocused LFM for experimental demonstrations by inserting a microlens array at the image plane of a normal wide-field microscope (Fig. 1a). A 4f system was used to relay the back focal plane of the microlens array at the image sensor, with each microlens covering approximately $13 \times 13$ sensor pixels (Methods). Once set up, the high-resolution 3D range of LFM was fixed with a specific objective. To flexibly adjust the volume size for different specimens, we applied a piezo stage for high-speed axial scanning at large axial steps ${ }^{9}$. With the matched numerical aperture (NA), LFM provides an extended depth of field $~ 169$-times larger than that of WFM, which makes it very different from other wide-field imaging methods. The fluorescence signals far from the focal plane exhibit similar Gaussian backgrounds in $\mathrm{WFM}^{23-25}$, while they show apparent distinguishable features between different angular components captured by LFM within a snapshot (Supplementary Fig. 1 and Supplementary Video 1). We find that these depth-dependent features provide LFM with a similar capability of computational optical sectioning as structured illumination microscopy ${ }^{31}$. The $3 \mathrm{D}$ out-of-focus fluorescence up to the centimeter scale can be reconstructed at low resolution, as long as we take it into consideration in the multislice model as a complete space. These layers far from the native objective plane have usually been neglected in previous methods due to the limited computational resources and contributed considerable background noises and artifacts in the high-resolution range (Fig. 1b). To address this problem, we propose a multiscale model in the phase-space domain by resampling the volume based on the nonuniform resolution of different axial planes, avoiding many unnecessary calculations in a complete space (Fig. 1b, Supplementary Fig. 2 and Methods). We then conducted a numerical 
simulation with gradually increasing background levels to show the significantly improved quantitative property of the multiscale model, which is barely considered in previous studies but important for biological analysis (Supplementary Fig. 3).

Except for out-of-focus fluorescence, the scattered photons pose another challenge in thick tissue, which cannot be rejected by optical sectioning due to the depthindependent property. Although multislice scattering models have achieved great success in the coherent imaging modalities ${ }^{32}$, they are barely considered in deconvolution algorithms for incoherent fluorescence imaging. As the 4D phase-space measurements can fully describe the partially coherent light-field distributions, they provide an opportunity to infer the native 3D fluorescence as well as the nonuniform 3D scattering coefficients. Here, we derived a multislice multiple-scattering model to differentiate the emission fluorescence and scattered photons based on the first Born approximation in the incoherent condition ${ }^{33}$ (Fig. 1c and Supplementary Note 1). Then, we applied the alternating direction method of multipliers (ADMM) algorithm ${ }^{34}$ with the multiscale model to update the volumetric fluorescence and 3D scattering potentials iteratively (Supplementary Fig. 2 and Supplementary Video 1).

By modeling both the out-of-focus and scattered photons, we retrieved the 3D fluorescence distribution quantitatively in thick tissue. To show the pipeline, we imaged a GFP-labeled tumor spheroid with a $63 \times / 1.4$ NA oil-immersion objective at an axial step of $10 \mu \mathrm{m}$ for 10 subvolumes. Strong background fluorescence was observed in the raw LF images, which was effectively removed by the capability of computational optical sectioning in QLFM (Fig. 1d). Each LF image has a specific axial range for highresolution 3D components. The large $3 \mathrm{D}$ volume with multiple axial steps was reconstructed as a whole during the iterations, making full use of the overlapped axial range and providing a better estimation of the background and uniform resolution at different axial planes (Methods and Supplementary Video 1). In contrast, traditional LFM 
125 shows strong artifacts due to crosstalk from the background. The axial side, with larger

126 out-of-focus photons, tends to have higher intensities, demonstrating the loss of the

127 quantitative property in thick tissue.

\section{Characterization of QLFM}

129 To quantitatively evaluate the improvement of QLFM, we imaged various 2- $\mu \mathrm{m}$

130 fluorescence beads embedded in a tissue-mimicking phantom made of intralipid and 131 agarose with a $40 \times / 1.0 \mathrm{NA}$ objective and calculated the average SBR at different 132 penetration depths (Methods, Fig. 2a, and Supplementary Fig. 4). By comparing with

133 WFM and different LFM models, shown in Fig. 2b, we found that the naive imaging 134 model of traditional LFM resulted in a similar performance as WFM in tissue penetration.

135 Our multiscale scattering model fully exploits the capability of LFM in deep-tissue 136 imaging with $20 \mathrm{~dB}$ SBR improvement over WFM. Such a great improvement facilitates

137 QLFM high-speed 3D imaging in thick tissue. We then imaged the same densely labeled 138 Drosophila brain by QLFM, traditional LFM, WFM, and confocal microscopy for 139 comparison (Supplementary Fig. 5). Despite reduced resolution due to the tradeoff

140 between spatial and angular resolutions in LFM, QLFM showed great sectioning 141 capability comparable to that of confocal microscopy without axial-scanning artifacts, 142 which was much better than those of traditional LFM and WFM.

Another problem hindering the quantitative reconstruction in LFM is the inaccurate estimation of the complicated high-dimensional PSF. As shown in Fig. 2c, the

145 system aberration will result in great system errors between the ideal PSF and the 146 experimental PSF measured with a sub-diffraction-limited fluorescence bead. We 147 propose a phase-retrieval-based algorithm to estimate the system aberration by iteratively 148 shrinking the disparity between the simulated PSF and the captured PSF along different 149 angular components with a single image (Supplementary Fig. 6). We find that the 
150 calibrated PSF with the aberration wavefront can greatly reduce the reconstruction 151 artifacts close to the native objective plane (Supplementary Fig. 6c). Numerical

152 simulations on different levels of aberrations also shows the effectiveness of the method

153 (Supplementary Fig. 7). We then characterized the system resolution by imaging 500-nm

154 fluorescence beads distributed in 1\% agarose with a $40 \times / 1.0$ NA water-immersion

155 objective. The average full width at half-maximum (FWHM) was used to estimate the

156 lateral and axial resolutions at different axial planes (Fig. 2d). In addition to the improved

157 resolution, QLFM with high-speed axial scanning at a step size of $30 \mu \mathrm{m}$ for 5 planes has

158 a uniform resolution of approximately $1.8 \times 1.8 \times 2.5 \mu \mathrm{m}^{3}$ across a large depth range 159 covering $\sim 330 \times 330 \times 180 \mu^{3}$. Moreover, as the multiscale model in phase-space domain 160 avoids the unnecessary up-sampling based on the effective resolution, it can reduce the 161 memory and computing time by orders of magnitude, especially for large depth ranges 162 during reconstruction to get rid of background (Fig. 2e). By modeling the same volume 163 range, our multiscale model with adaptive sampling rate shows the same performance as 164 traditional methods, but reduces the computing time from several hours to several 165 seconds, on a desktop computer equipped with a normal graphical processing unit (CPU: 166 Intel i9-9980XE, RAM: 128 GB, GPU: NVIDIA GeForce RTX 2080 Ti), facilitating the 167 practical use of QLFM.

168 High-speed 3D imaging of Drosophila embryo and Zebrafish larvae

169 To demonstrate the superior performance of QLFM over previous methods using compact 170 systems, we performed in vivo imaging of various fast biological dynamics in different 171 specimens. First, we imaged the whole-embryo development of histone-labeled 172 Drosophila at the millisecond scale with both $40 \times / 1.0$ NA (Figs. 3a and b) and 20×/0.5 173 NA (Figs. 3c and d) objectives for comparison. Different from previous methods 174 capturing only part of the volume at a time ${ }^{35}$, LFM achieves better photon efficiency with 175 low phototoxicity by illuminating and detecting the entire volume simultaneously. While 
176 there was almost no contrast in traditional LFM due to the dense labeling and strong 177 scattering, QLFM could still distinguish single cells deep in the embryo at the millisecond 178 scale, extending the applications of LFM to developmental biology.

Second, we performed in vivo imaging of heart-beating dynamics in zebrafish

180 larvae, which are difficult to fully capture without simultaneous exposure of the whole volume at high speed. Traditional LFM shows low resolution and contrast with severe artifacts (Fig. 4a), but QLFM, with a more accurate computational model, could obtain artifact-free high-resolution 3D volumes without the requirement of additional light-sheet illumination and multiview objectives (Figs. 4b and c, and Supplementary Figs. 8 and 9). We found successive improvement with increasing model complexity, indicating the necessity of various factors in the model, including out-of-focus fluorescence, sample scattering, and system aberrations (Supplementary Video 2).

Whole-brain neural recording in zebrafish larvae is another typical application of LFM, but the imaging performance is far from satisfactory due to the densely packed neurons without complicated systems ${ }^{14}$ (Fig. 4d). Although the spatiotemporal sparsity

191 facilitates the accurate localization and extraction of neural signals ${ }^{36,37}$, it is still 192 susceptible to nonuniform background fluorescence and inevitable structural changes in 193 living animals. By imaging the whole-brain neural activities in zebrafish larvae at $24 \mathrm{~Hz}$ 194 with a $20 \times / 0.5$ NA objective, we demonstrate that QLFM can achieve effective singleneuron resolution with proper models to remove both out-of-focus and scattered photons

196 for each single frame without the requirement to calculate the standard deviation of many

197 frames for better contrast (Figs. 4e and f, and Supplementary Video 3). The temporal 198 traces of several neurons with simple region-of-interest (ROI) averaging demonstrate the 199 increased contrast and reduced crosstalk between adjacent neurons in QLFM (Fig. 4g). 


\section{Large-scale 3D calcium imaging in mice brain}

The mammalian brain is a more challenging case due to its strong scattering and dense neural structures. We tested the performance of QLFM by imaging the 3D calcium activities in an awake head-fixed mouse, which was labeled with GCaMP6s by virus injection, under a $20 \times / 0.5 \mathrm{NA}$ objective. To visualize the $3 \mathrm{D}$ distribution of neurons, traditional LFM usually requires the standard deviation of thousands of frames to reduce the background (Supplementary Fig. 10a). However, the 3D signals of a single frame are usually flooded in the background fluctuations due to the low SBR (Fig. 5a). In contrast, with the capability of computational optical sectioning, QLFM shows significantly improved contrast (Fig. 5b and Supplementary Video 4). In addition to the detection of

211 more neurons (Supplementary Fig. 10a), QLFM allows the measurement of calcium

212 dynamics in a quantitative manner without the need for additional background

213 subtractions or filtering to improve the contrast, making it more reliable for large-scale

214 neural recording (Fig. 5c). Background fluorescence usually increases with the increasing 215 of NA due to the smaller depth of field, especially for the wide-field imaging. We 216 therefore imaged the same mouse with a $40 \times / 1.0 \mathrm{NA}$ water-immersion objective at 217 different depths (Supplementary Figs. 10b-e and Supplementary Video 5). QLFM not 218 only resolved more neurons with better resolution and contrast than traditional LFM but

219 also showed stable performance at different depths up to $300 \mu \mathrm{m}$ (Figs. $5 \mathrm{~d}$ and e). redundant 3D imaging speed for calcium dynamics. We then conducted millisecond-scale calcium imaging in awake double-transgenic Rasgrf2-2A-dCre/Ai148D mice across a large volume covering $\sim 700 \times 700 \times 180 \mu \mathrm{m}^{3}$ by high-speed scanning of 3 planes. While there was barely contrast in traditional LFM due to the severe artifacts especially in the axial domain, which was similar to previous work ${ }^{36}$, QLFM showed a much larger 
226 penetration depth with uniform resolution (Figs. $5 \mathrm{f}$ and $\mathrm{g}$ and Supplementary Video 6).

227 Even the neurons located $\sim 400 \mu \mathrm{m}$ deep exhibited remarkable calcium responses.

\section{Discussion}

229 In summary, we developed an incoherent multiscale scattering model to fully exploit the

230 intrinsic high-dimensional property of phase-space measurements and achieved 231 computational optical sectioning, facilitating high-speed, large-scale, quantitative 3D 232 imaging in deep tissue with a compact system. provides a tomographic framework by keeping the photons focused along different angles with an extended depth of field. Such a process makes the signals originated from different axial planes show much more distinguishable features than WFM, which can be utilized to remove background fluorescence far from the native objective plane computationally, as long as we model the imaging process in a complete space. Additional confocal rejection or light-sheet illuminations will definitely further reduce the shot-noise fluctuations by getting rid of the background photons physically but at the cost of system compactness, space constraints, or detection parallelization.

In addition, as a novel computational model, our method is compatible with

243 different phase-space imaging schemes without the requirement of any hardware modifications. In combination with the low phototoxicity and high photon efficiency, the orders-of-magnitude reductions in background fluorescence, artifacts, and computational costs enable the practical and versatile applications of QLFM as a compact and robust add-on to normal wide-field microscopy, making the advanced imaging capability generally accessible to the broad biology community. 


\section{Methods}

\section{Experimental setup}

252 Our QLFM system works as an add-on to a normal epifluorescence microscope, with a 253 microlens array inserted at the image plane. A customized upright microscope was used 254 for mouse experiments, while a commercial inverted microscope (Zeiss Observer Z1) was used for the others. Another $4 \mathrm{f}$ system (with a magnification of 0.845 ) relayed the back 256 focal plane of the microlens array (with a pitch size of $100 \mu \mathrm{m}$ and focal length of 2.1 $257 \mathrm{~mm}$ ) to the camera (Andor Zyla 4.2 plus, 2,048 $\times 2,048$ pixels) so that each microlens 258 covered $\sim 13 \times 13$ sensor pixels, corresponding to a $2.15 \mu \mathrm{m} \times 2.15 \mu \mathrm{m}$ area at the sample 259 plane for the $40 \times / 1.0$ NA water-immersion objective. Multiple lasers (488 nm and 561 $260 \mathrm{~nm}$ ) were used for the fluorescence excitation of multiple channels, which were synchronized with the camera for time division multiplexing. A piezo objective scanner

262 (PI P-725.4CD) was used for high-speed axial scanning with a resolution of $1.25 \mathrm{~nm}$ at $100 \mathrm{~Hz}$. Detailed imaging conditions and reconstruction parameters for all fluorescence experiments in the paper, including the excitation power, exposure time, frame rate, voxel size, fluorophore, protein, filter set, and objective, are illustrated in Supplementary Table 1.

\section{D deconvolution with a multiscale scattering model}

269 To reconstruct the 3D fluorescence information in deep tissue in a quantitative way, we

270 propose a novel 3D deconvolution algorithm with a multiscale scattering model to 271 iteratively update both the emission fluorescence and scattering photons across an 272 extremely large depth range (Fig. 1c). The whole pipeline of the algorithm with the 273 pseudocode is shown in Supplementary Fig. 2.

With simple pixel realignment, the raw LF data can be represented as multiple angular components, or smoothed phase-space measurements ${ }^{20}$, which can be used for 
276 phase-space deconvolution ${ }^{29}$ to reduce artifacts and increase the convergence speed.

277 However, the reconstructed volume, regardless of the deconvolution algorithm ${ }^{29,30}$, is

278 limited to only dozens of axial planes due to the heavy computational cost. Therefore, the

279 out-of-focus fluorescence has usually been modeled as a constant in previous methods,

280 which is fine for thin samples but leads to severe artifacts and background noise in deep

281 tissue with nonuniform out-of-focus distributions. Fortunately, both the lateral and axial

282 resolution of LFM will gradually decrease with increasing distances from the native

283 objective plane. We can establish a multiscale grid to sample a large 3D volume at

284 different intervals based on the characterized resolution with an exponential fit

285 (Supplementary Fig. 2). The out-of-focus fluorescence with a nonuniform 3D distribution

286 can then be estimated with the depth-dependent features within a large depth of field

287 (Supplementary Fig. 1), akin to structured illumination microscopy exhibiting

288 computational optical sectioning. As this method does not require dense axial sampling

289 and a lateral sampling rate as high as the camera pixel number for each plane, which was

290 necessary in previously reported methods due to the spatially variant $\mathrm{PSF}^{30}$, the unknown

291 variables can be reduced by orders of magnitude to accelerate the reconstruction speed

292 when modeling the out-of-focus fluorescence of a much larger volume (Fig. 2e). For the

293 same volume size, our multiscale model can achieve almost the same performance as

294 traditional dense-sampling models at a much faster speed. In contrast, the traditional

295 method $^{29}$, without a sufficient axial range in the model, shows apparent artifacts and an

296 increase in the background.

297 Scattering is a recurring challenge in thick tissue and is the fundamental limitation

298 of the penetration depth in light microscopy, leading to reduced resolution and contrast $\mathrm{t}^{38}$.

299 Although the multilayer scattering model has recently achieved great success in coherent

300 imaging modalities, such as diffraction tomography ${ }^{39}$, the spatially nonuniform influence

301 of scattering is barely considered in fluorescence imaging. LFM provides a great opportunity to differentiate emission fluorescence and scattered photons with its 4D 
304 Born approximation in incoherent conditions by describing the relation between the

$$
I^{(s)}(\mathbf{r})=\frac{1}{2} \int_{V} F\left(\mathbf{r}^{\prime}\right) I^{(i)}\left(\mathbf{r}^{\prime}\right) \frac{1}{\left\|\mathbf{r}-\mathbf{r}^{\prime}\right\|_{2}^{2}} \mathrm{~d}^{3} r^{\prime}
$$

307 where $F$ is defined as the scattering potential energy at the $3 \mathrm{D}$ position $r$, and $V$ is the

308 whole 3D volume range. The detailed derivations and discretized version are illustrated

309 in Supplementary Note 1 . Then, an ADMM framework ${ }^{34}$ is used to iteratively update the

310 sample information and the scattered photons to retrieve the quantitative fluorescence

311 distributions in deep tissue with increased contrast and resolution (Supplementary Fig.

312 2). The reconstruction time of a single volume with a full FOV of the sensor used here is

313 about several seconds for a normal desktop computer with a graphical processing unit.

\section{3D deconvolution with axial scanning for LFM}

315 The high-resolution 3D range of LFM for a snapshot is fixed for a specific objective.

316 High-speed axial scanning at a large step size is a straightforward method to flexibly

317 adjust the depth range and 3D imaging speed. Such a capability is important for versatile

318 applications with different requirements for the volume size and imaging speed.

319 However, traditional methods show severe artifacts at the stitching edges at low contrast

320 (Figs. 1d and 5f), as they usually reconstruct each subvolume based on every single LF

321 image separately and stitch the subvolumes in the axial domain ${ }^{36}$. Here, with the

322 multiscale model in QLFM, we take the entire 3D volume as a whole with the multiscale

323 sampling rate during reconstruction. As shown in Supplementary Video 1, after pixel

324 realignment of all the raw LF images, we can achieve multiple focal stacks for different

325 angular components, making full use of the intrinsic continuous property of different

326 angular components in the axial domain. Due to the large depth of field for each angular

327 component in QLFM, we can use a large step for axial scanning with much less time 
required to cover the same volume than in WFM. Then we update the entire large volume as a whole along different angles, viewing each angular focal stack as the minimum unit

330 to calculate the error map for each iteration. Such a process is akin to wide-field

331 deconvolution first for each angle followed by tomographic reconstruction later for different angles. Finally, the same ADMM framework is applied to the entire volume to update the emission fluorescence and scattered photons iteratively. In this case, uniform resolution can be achieved at different axial planes across a large depth range without any artifacts (Fig. 1d and 2d). In addition, the computational cost can be further reduced, as we can conduct 3D deconvolution with the phase-space PSF for each angular focal stack.

\section{Phase-retrieval-based PSF calibration}

338 The PSF of traditional LFM is calculated based on wave optics theory in an ideal imaging 339 condition $^{29,30}$. However, the experimental imaging system usually has complicated system aberrations, which will not only reduce the imaging resolution but also introduce severe reconstruction artifacts close to the native objective plane. Here, we propose a phase-retrieval-based algorithm to calibrate the experimental PSF with a single image of subdiffraction-limited fluorescence beads (Supplementary Fig. 6). The simulated PSF is

344 first initialized with experimental parameters without any aberration wavefront. Then, we

345 calculate the correlations between the captured image and the simulated PSF along 346 different angular components to estimate the wavefront error at different sub-apertures.

347 The aberrated wavefront of the whole NA is then integrated from the correlation map for 348 continuous distributions. The estimated wavefront is fitted with Zernike polynomials and

349 fed into the wave optics model to generate the new simulated PSF, which is used again 350 to match with the captured PSF. The calibrated PSF is iteratively updated through the 351 above process until the phase map converges, which usually takes approximately 3 4

352 iterations. Interestingly, we found that the experimental system with spherical aberrations 353 can remove the reconstruction artifacts close to the native objective plane as long as we had an accurate estimation of the aberration wavefront. 


\section{Fluorescence bead preparation for system characterization}

357 For resolution characterization, 500-nm yellow-green fluorescent microspheres (Thermo

358 Scientific, FluoSpheres, carboxylate-modified microspheres) were mixed with 1\% agarose at a ratio of 1:1,000,000. We placed the mixture in a $35-\mathrm{mm}$ petri dish and captured $\sim 100$ LF images for statistical analysis with a 40×/1.0 NA water-immersion objective. We then calculated the FWHM of the lateral and axial profiles of the reconstructed beads on different axial planes. For each block covering $10 \mu \mathrm{m}$, we chose 10 beads with the highest fitting degree to calculate the mean and standard deviation.

For SBR characterization, we fabricated a scattering phantom with the mixture of $1 \%$ agarose, $1 \%$ intralipid (Absin 68890-65-3, 20\% emulsion), and 0.025\% 2- $\mu \mathrm{m}$ fluorescence beads, which was placed in a $35-\mathrm{mm}$ petri dish. The $2-\mu \mathrm{m}$ fluorescence beads (Thermo Scientific, FluoSpheres, carboxylate-modified microspheres) were randomly distributed in the intralipid and imaged using a 40×/1.0 NA water-immersion objective. The reconstructed mean intensity of the beads was viewed as the signal, while the mean intensity of the reconstructed background without samples was viewed as the background. Several reconstructed slices at different imaging depths are shown in Supplementary Fig. 4.

\section{Tumor spheroid preparation}

374 B16 cells (ATCC® CRL-6475 ${ }^{\mathrm{TM}}$, mouse skin melanoma cells) were purchased from

375 ATCC and cultured in RPMI 1640 medium supplemented with 10\% FBS, 1\% pen/strep 376 antibiotics and 1\% NEAA (all from GIBCO). Cells were then transfected with the EGFP-

377 PH plasmid (Addgene Plasmid \#96948), and stable EGFP-expressing B16 cells were 378 selected by neomycin (G418) and maintained. To prepare tumor spheroids, $4 \times 10^{3}$ EGFP379 expressing B16 cells per well were seeded in round-bottomed 96-well plates (Corning) and cultured in RPMI 1640 medium supplemented with 10\% FBS, 2\% B-27 supplement 
381 (GIBCO), 2\% methyl cellulose (Sigma-Aldrich), 1\% pen/strep antibiotics and 1\% NEAA.

382 After 2 days, each formed spheroid was transferred as 1 spheroid per well and cultured

383 for another 2 days. During LFM imaging, the GFP-B16 tumor spheroids were transferred

384 to Lab-Tek II cover-glass-bottomed 8-chamber slides and imaged in HBSS supplemented with $2 \%$ FBS (all from Invitrogen) using a $63 \times / 1.4$ NA oil-immersion objective.

\section{In vivo imaging of zebrafish larvae}

387 All zebrafish experimental procedures were conducted with ethical approval from the

388 Animal Care and Use Committee of Tsinghua University. For imaging of the vasculature 389 and blood flow dynamics, Tg(flk:EGFP; gatal:DsRed) transgenic zebrafish embryos 390 were collected and cultured at $28.5{ }^{\circ} \mathrm{C}$ in Holtfreter's solution. At 4-5 days

391 postfertilization (dpf), the zebrafish larvae were anesthetized by ethyl 3-aminobenzoate 392 methanesulfonate salt $(100 \mathrm{mg} / \mathrm{L})$ and mounted in $1 \%$ low-melting-point agarose for 393 imaging at $26-27^{\circ} \mathrm{C}$. For whole-brain calcium imaging, $\operatorname{Tg}($ huc:GCaMP6) transgenic 394 zebrafish embryos were collected and kept at $28.5^{\circ} \mathrm{C}$ in Holtfreter's solution. At 4-5 dpf, 395 the larvae were mounted in $1 \%$ low-melting-point agarose for imaging at $26-27^{\circ} \mathrm{C}$.

\section{Preparation of fixed Drosophila brain samples}

398 All Drosophila experimental procedures were conducted with ethical approval from the

399 Animal Care and Use Committee of Tsinghua University. Female Drosophila brains were 400 dissected and fixed in 4\% paraformaldehyde (PFA, Cat\# AR-0211, Dingguo Biotech, 401 China) at room temperature for 30 mins on a shaker. Each brain was rehydrated with $4020.3 \%$ Triton X-100 (Solarbio 524A0513) in phosphate-buffered saline (PBS) for $4 \times 20$ 403 mins at room temperature and then incubated in block solution (5\% goat serum in washing 404 buffer) for 30 mins at room temperature. The brain was then incubated overnight with 405 primary antibody (mouse monoclonal nc82 (Developmental Studies Hybridoma Bank)), 406 which was diluted at 1:500 in block solution at $4{ }^{\circ} \mathrm{C}$. The brain was then washed in $0.5 \%$ 
407 PBST for $3 \times 1$ hour at room temperature. Finally, the brain was mounted directly for 408 imaging by LFM.

\section{Imaging of Drosophila embryos}

410 All Drosophila embryos (Fig. 3) expressed histones tagged with EGFP (His2Av, 411 BL24163). The collection and preparation of Drosophila embryos were performed 412 according to the commonly used protocol ${ }^{40}$. Two-hour Drosophila embryos were 413 collected within a specific collection chamber. After incubation at $25^{\circ} \mathrm{C}$ for 10 hours, 414 each embryo was attached to a glass microscope slide with double-sided sticky tape. We 415 used forceps to carefully roll the embryo on the tape until its chorion popped open. Then, 416 the embryo was transferred to the glue line on a dish (Ibidi $\mu$-Dish, $35 \mathrm{~mm}$, high) and

417 covered with mineral oil (Sigma-Aldrich, Halocarbon 27 oil) for live imaging.

\section{Mouse experiments}

419 All procedures involving mice were approved by the Institutional Animal Care and Use 420 Committee of Tsinghua University. We used both male and female C57BL/6 mice 10 421 weeks to 6 months old without randomization or blinding. We performed the craniotomy 422 as previously described ${ }^{41}$, with a window size of $\sim 8 \mathrm{~mm} \times 8 \mathrm{~mm}$. Then, we installed a flat 423 optical window and cemented a custom-made coverslip (D-shape) and aluminum head 424 posts to the skull. For acute imaging, we used adult double-transgenic Rasgrf2-2A$425 \mathrm{dCre} / \mathrm{Ai148D}$ mice (JAX No.: 022864 and 030328) to specifically label cortical layer 2/3 426 neurons $^{42}$. For chronic imaging, adult C57BL/6 mice injected with diluted AAV9-hSyn427 GCaMP6s virus (from BrainVTA Technology Co., Ltd., China) were allowed to recover 428 for at least 2 weeks after craniotomy. During LFM imaging, awake mice were placed in 429 a tube with the head restrained under the objective.

\section{Data analysis}


431 All data analyses were performed with customized MATLAB (MathWorks, MATLAB

432 2018) programs and Amira (Thermo Fisher Scientific, Amira 2019). The hardware was

433 controlled using LabVIEW 2018 (National Instruments). The 3D rendering of the

434 volumes in figures and videos was performed by Amira. The 3D tracking of 7 435 representative blood cells in the heart of the zebrafish larvae was carried out manually in

436 MATLAB.

\section{Neural activity extraction}

439 The calcium responses in both zebrafish and mice were extracted directly through signal 440 averaging of the manually selected ROIs covering the selected neurons. The ROIs were $441 \sim 8 \times 8 \times 10 \mu \mathrm{m}^{3}$ in size for zebrafish larvae and $\sim 10 \times 10 \times 10 \mu \mathrm{m}^{3}$ in size for mice to match 442 the size of the neuron. The temporal traces of neural activity were calculated by $\Delta F / F_{0}=$ $443\left(F-F_{0}\right) / F_{0}$, where $F$ is the raw averaged intensity of the ROI, and $F_{0}$ is the baseline 444 fluorescence intensity. To estimate $F_{0}$ for each ROI, we first calculated the average 445 intensity of the trace and averaged all time points with signals below $120 \%$ of the calculated average.

\section{Data availability}

449 All relevant data are available from the corresponding authors upon reasonable request.

\section{Code availability}

452 We will open all of codes with example datasets in Google Drive and Github after the 453 paper is accepted.

\section{Acknowledgments}

456 We thank Li Yu, Jiesi Feng, Yulong Li, Jing He, Guihua Xiao, and Hao Xie for their 457 assistance in sample preparation. This work was supported by the National Natural 458 Science Foundation of China (61327902, 61827804, 61620106005), the Beijing 
460 National Key Research and Development Program of China (2020AAA0130000), the

461 Postdoctoral Science Foundation of China (2019M660644), and the Tsinghua University

462 Initiative Scientific Research Program. J.W. sincerely appreciates funding from the

463 National Postdoctoral Program for Innovative Talent.

464

465

\section{Author contributions}

466

Q.D., X.J., and J.W. conceived and designed the project. Q.D. and X.J. supervised the research. Z.L. and J.W. designed and built the optical system. Y.Z., X.J. and J.W. conducted the numerical simulation and developed the deconvolution algorithm with the multiscale scattering model. J.W. and D.J. designed the biological experiments. X.L. conducted the hardware synchronization. J.X. derived the scattering model in incoherent

472 the zebrafish larvae. Z.L., Y.Z., D.J. and J.W. conducted most biological experiments,

473 data collection and volume reconstructions. X.L. and Y.C. conducted algorithm 474 performance optimization. Y.Z. designed the system calibration method. J.W., Z.L., Y.Z., 475 X.J., and Q.D. prepared figures and wrote the manuscript with input from all authors.

\section{Competing financial interests}

478 The authors declare no competing financial interests.

Materials \& correspondence

481 Correspondence and requests for materials should be addressed to 


\section{References}

1. Weisenburger, S. \& Vaziri, A. A guide to emerging technologies for large-scale and whole-brain optical imaging of neuronal activity. Annu. Re. Neurosci. 41, 431452 (2018).

2. Ji, N., Freeman, J. \& Smith, S. L. Technologies for imaging neural activity in large volumes. Nat. Neurosci. 19, 1154 (2016).

3. Pittet, M. J. \& Weissleder, R. Intravital imaging. Cell 147, 983-991 (2011).

4. Pawley, J.B. Handbook of Biological Confocal Microscopy, edn 2. (Plenum Press, New York, 1995).

5. Shimozawa, T. et al. Improving spinning disk confocal microscopy by preventing pinhole cross-talk for intravital imaging. Proc. Natl. Acad. Sci. U. S. A. 110, 33993404 (2013).

6. Helmchen, F. \& Denk, W. Deep tissue two-photon microscopy. Nat. Methods 2, 932-940 (2005).

7. Zhu, G., van Howe, J., Durst, M., Zipfel, W. R. \& Xu, C. Simultaneous spatial and temporal focusing of femtosecond pulses. Opt. Express 13, 2153-2159 (2005).

8. Bouchard, M. B. et al. Swept confocally-aligned planar excitation (SCAPE) microscopy for high-speed volumetric imaging of behaving organisms. Nat. Photonics 9, 113-119 (2015).

9. Ahrens, M. B., Orger, M. B., Robson, D. N., Li, J. M. \& Keller, P. J. Whole-brain functional imaging at cellular resolution using light-sheet microscopy. Nat. Methods 10, 413-420 (2013).

10. Wu, Y. \& Shroff, H. Faster, sharper, and deeper: structured illumination microscopy for biological imaging. Nat. Methods 15, 1011-1019 (2018).

11. Mertz, J. Optical sectioning microscopy with planar or structured illumination. Nat. Methods 8, 811-819 (2011).

12. Winter, P.W. \& Shroff, H. Faster fluorescence microscopy: advances in high speed biological imaging. Curr. Opin. Chem. Biol. 20, 46-53 (2014).

13. Laissue, P. P. et al. Assessing phototoxicity in live fluorescence imaging. Nat. Methods 14, 657-661 (2017).

14. Prevedel, R. et al. Simultaneous whole-animal 3D imaging of neuronal activity using light-field microscopy. Nat. Methods 11, 727-730 (2014).

15. Alonso, M. Wigner functions in optics: describing beams as ray bundles and pulses as particle ensembles. Adv. Opt. Photon. 3, 272-365 (2011).

16. Waller, L., Situ, G. \& Fleischer, J. W. Phase-space measurement and coherence synthesis of optical beams. Nat. Photon. 6, 474-479 (2012).

17. Liu HY, Jonas E, Tian L, Zhong JS, Recht B et al. 3D imaging in volumetric scattering media using phase-space measurements. Opt. Express 2015; 23: 1446114471.

18. Takasaki, K. T. \& Fleischer, J. W. Phase-space measurement for depth-resolved memory-effect imaging. Opt. Express 22, 31426-31433 (2014).

19. Levoy, M., Ng, R., Adams, A., Footer, M. \& Horowitz, M. Light field microscopy. ACM Trans. Grapgh. 25, 924-934 (2006).

20. Tian, L., Zhang, Z., Petruccelli, J. C. \& Barbastathis, G. Wigner function measurement using a lenslet array. Opt. Express 21, 10511-10525 (2013).

21. Wagner, N. et al. Instantaneous isotropic volumetric imaging of fast biological processes. Nat. Methods 16, 497-500 (2019). 
22. Lin, Q. et al. Cerebellar Neurodynamics Predict Decision Timing and Outcome on the Single-Trial Level. Cell 180, 536-551 (2020).

23. Abrahamsson, S. et al. Fast multicolor 3D imaging using aberration-corrected multifocus microscopy. Nat. Methods 10, 60-63 (2013).

24. Yang, W. et al. Simultaneous Multi-plane Imaging of Neural Circuits. Neuron 89, 269-284 (2016).

25. Xiao, S. et al. High-contrast multifocus microscopy with a single camera and zsplitter prism. Optica 7, 1477-1486 (2020).

26. Taylor, M. A., Nöbauer, T., Pernia-Andrade, A., Schlumm, F. \& Vaziri, A. Brainwide 3D light-field imaging of neuronal activity with speckle-enhanced resolution. Optica 5, 343-353 (2018)

27. Wagner, N. et al. Instantaneous isotropic volumetric imaging of fast biological processes. Nat. Methods 16, 497-500 (2019).

28. Zhang, Z. et al. Imaging volumetric dynamics at high speed in mouse and zebrafish brain with confocal light field microscopy. Nat. Biotechnol. 1-10 (2020).

29. Lu, Z. et al. Phase-space deconvolution for light field microscopy. Opt. Express 27, 18131-18145 (2019).

30. Broxton, M. et al. Wave optics theory and 3-D deconvolution for the light field microscope. Opt. Express 21, 25418-25439 (2013).

31. Gustafsson, M. G. L. Nonlinear structured-illumination microscopy: Wide-field fluorescence imaging with theoretically unlimited resolution. Proc. Natl. Acad. Sci. U.S.A. 102, 13081-13086 (2005).

32. Tian, L. \& Waller, L. 3D intensity and phase imaging from light field measurements in an LED array microscope. Optica 2, 104-111 (2015).

33. Born, M., Wolf, E. Principles of Optics: Electromagnetic Theory of Propagation, Interference and Diffraction of Light. Elsevier (2013).

34. Boyd, S., Parikh, N., Chu, E., Peleato, B. \& Eckstein, J. Distributed optimization and statistical learning via the alternating direction method of multipliers.

Foundations and Trends in Machine Learning 3, 1-122 (2010).

35. Royer, L. A. et al. Adaptive light-sheet microscopy for long-term, highresolution imaging in living organisms. Nat. Biotechnol. 34, 1267-1278 (2016).

36. Nöbauer, T. et al. Video rate volumetric Ca2+ imaging across cortex using seeded iterative demixing (SID) microscopy. Nat. Methods 14, 811 (2017).

37. Pégard, N. C. et al. Compressive light-field microscopy for 3D neural activity recording. Optica 3, 517-524 (2016).

38. Theer, P. \& Denk, W. On the fundamental imaging-depth limit in two-photon microscopy. J. Opt. Soc. Am. A 23, 3139-3149 (2006).

39. Chen, M., Ren, D., Liu, H.-Y., Chowdhury, S. \& Waller, L. Multi-layer Born multiple-scattering model for 3D phase microscopy. Optica 7, 394-403 (2020).

40. Conduit, P. T., Hayward, D. \& Wakefield, J. G. Microinjection techniques for studying centrosome function in Drosophila melanogaster syncytial embryos. Methods Cell Biol. 129, 229-249 (2015).

41. Chen, T. W. et al. Ultrasensitive fluorescent proteins for imaging neuronal activity. Nature 499, 295-300 (2013).

42. Daigle, T. L. et al. A Suite of Transgenic Driver and Reporter Mouse Lines with Enhanced Brain-Cell-Type Targeting and Functionality. Cell 174, 465-480 (2018). 
a
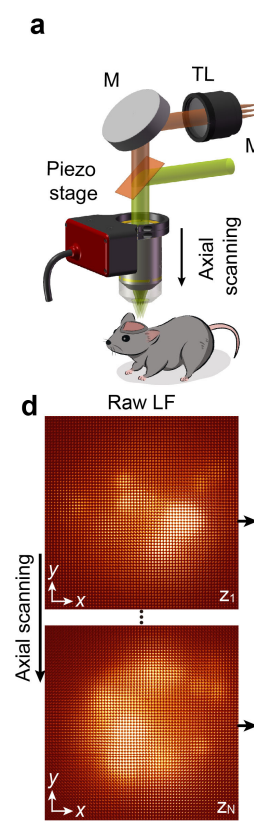

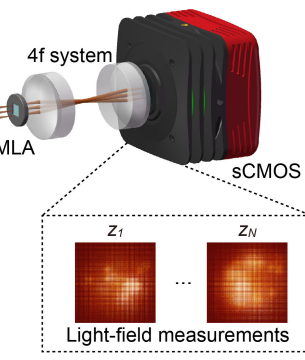

b

Incomplete space

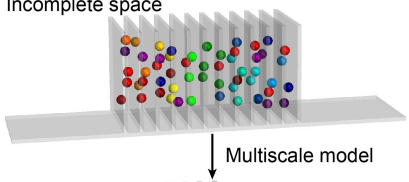

Multiscale model

Complete space

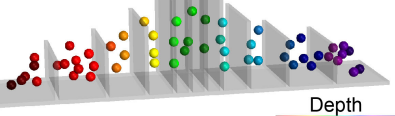

Depth

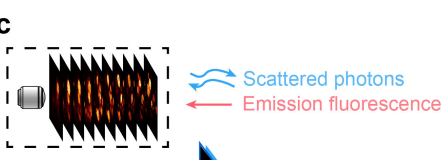

Computational optical sectioning
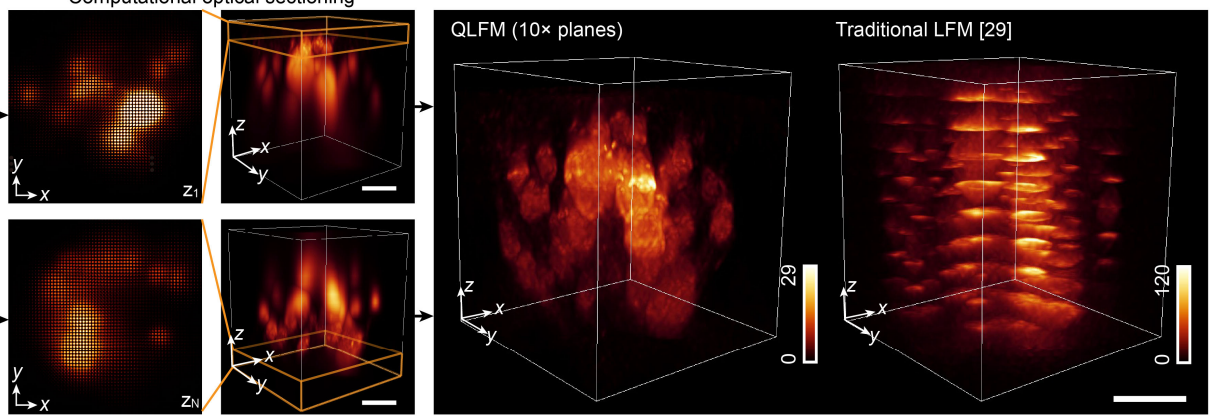

Fig. 1 | Schematic of quantitative light-field microscopy (QLFM). a, Experimental

setup of our QLFM system with a simple microlens array (MLA) inserted at the image plane for snapshot phase-space measurements. A piezo stage is used for high-speed axial scanning at a large step size periodically. b, Concept of the multiscale model. Incomplete space used in traditional LFM reconstruction results in strong background noise and loss of quantitative properties in complicated environments. We apply different sampling rates in 3D based on the effective resolution of LFM at different axial planes to model a large volume for background rejection with an orders-of-magnitude reduction in computational cost. c, Schematic of the multiscale scattering model. We differentiate the multiplescattered photons from native emission fluorescence in the multislice model based on the first Born approximation to retrieve 3D fluorescence quantitatively in deep tissue. d,

592 Illustrations of the algorithm by imaging GFP-labeled B16 tumor spheroids. Due to the extended depth of field, even the out-of-focus fluorescence far from the native objective plane has apparent depth-dependent features in LFM, which can be reconstructed in the multiscale model for computational optical sectioning. The axially scanned LF images of

59610 planes can be realigned into multiple angular focal stacks to reconstruct the entire 597 volume as a whole without artifacts. QLFM shows greatly improved resolution and 598 contrast over traditional LFM which reconstructs each subvolume separately. Scale bars, $30 \mu \mathrm{m}$. 
600

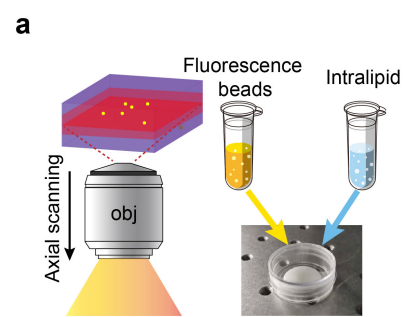

d

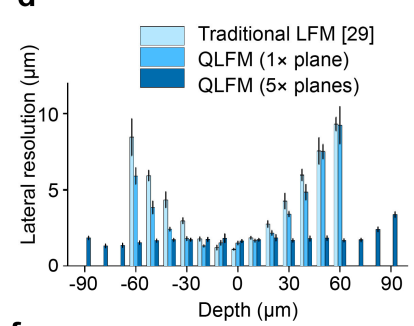

f

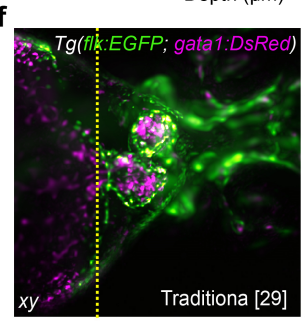

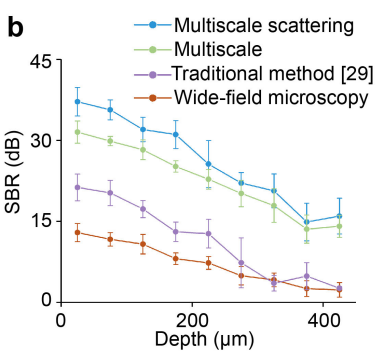

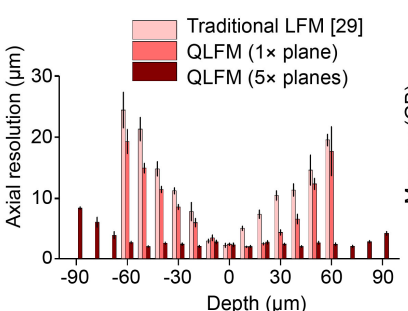

Depth $(\mu \mathrm{m})$
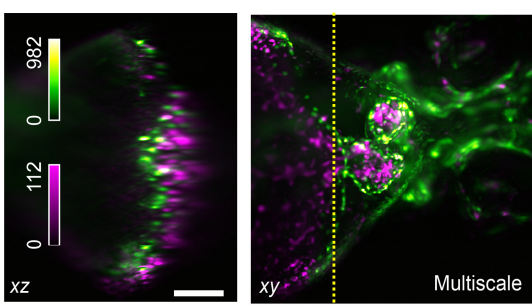
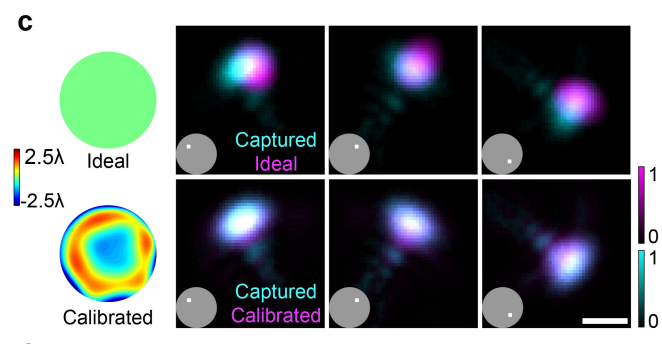

e
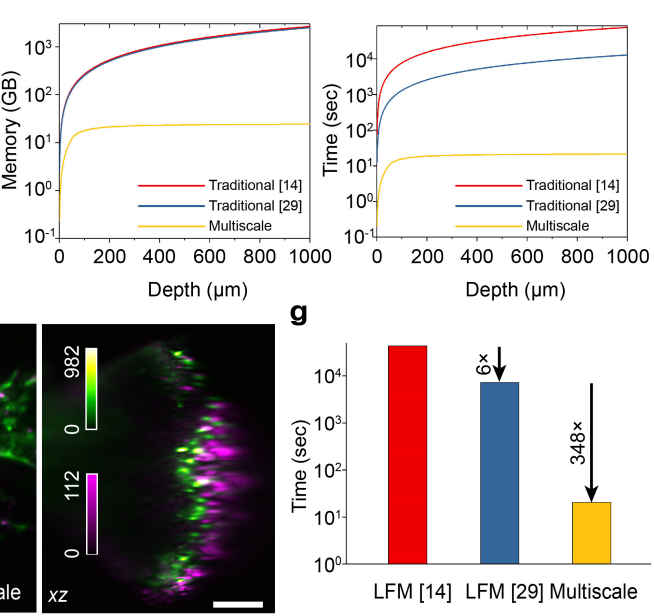

Fig. 2 | Characterization of quantitative light-field microscopy (QLFM). a,

Illustrations of the SBR characterization experiment. We fabricated the scattering

604 phantom with the mixture of $0.025 \% 2-\mu$ m fluorescence beads, $1 \%$ intralipid, $1 \%$ agarose

605 in a petri dish. Then we imaged the sample with a 40×/1.0NA water-immersion objective

606 at different penetration depths with WFM and LFM. b, SBR curves of the fluorescence

607 beads at different penetration depths in the intralipid-based tissue-mimicking phantom

608 obtained by WFM, traditional LFM, QLFM with the multiscale model only, and QLFM

609 with the multiscale scattering model. We chose 10 fluorescence beads with the highest

610 fitting degrees for each block covering approximately $40 \mu \mathrm{m}$. QLFM shows $\sim 20 \mathrm{~dB}$

611 improvement in the SBR over WFM, indicating an improved penetration depth in deep

612 tissue. c, Comparisons among the experimental PSF, the ideal PSF without aberrations,

613 and the calibrated PSF of several selected angular components marked in the inset under

614 the $40 \times / 1.0$ NA water-immersion objective. The calibrated wavefront of the system

615 aberration estimated by our iterative phase-retrieval algorithm is shown on the left. d,

616 Lateral and axial resolution at different axial planes in traditional LFM and QLFM with

6171 and 5 axially scanned planes, which is estimated by the FWHM of subdiffraction-

618 limited fluorescence beads. For each block covering $10 \mu \mathrm{m}$, we chose 10 fluorescence 
619 beads with the highest fitting degree for statistical analysis. e, The curves of the required

620 memory and computation time versus the reconstructed depth range for each volume by

621 different methods with a $20 \times / 0.5 \mathrm{NA}$ objective, indicating that the multiscale model is

622 essential to cover a large depth of range for computational optical sectioning. f,

623 Orthogonal MIPs of the beating heart in zebrafish larvae reconstructed by different

624 methods with a large axial range. The multiscale model shows similar performance as

625 traditional methods with orders-of-magnitude reduction in computational costs. $\mathbf{g}$, The

626 bar graph shows the time required by different methods to reconstruct the volumes shown

627 in f, covering about $\sim 700 \times 700 \times 560 \mu \mathrm{m}^{3}$. Scale bars, $5 \mu \mathrm{m}$ (c) and $100 \mu \mathrm{m}(\mathbf{f})$. 

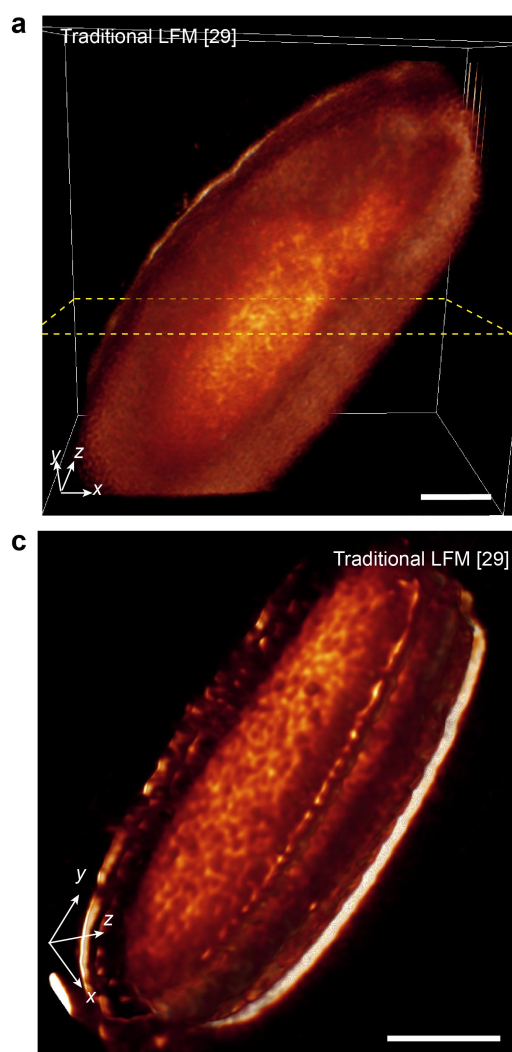

d

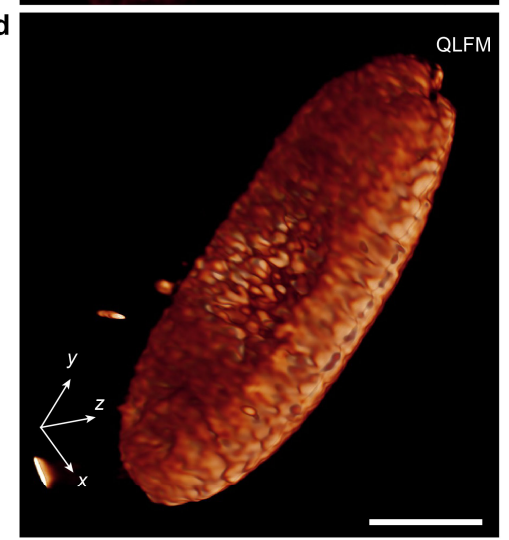

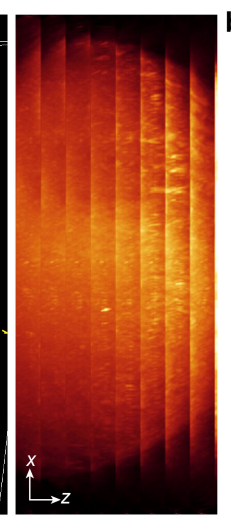
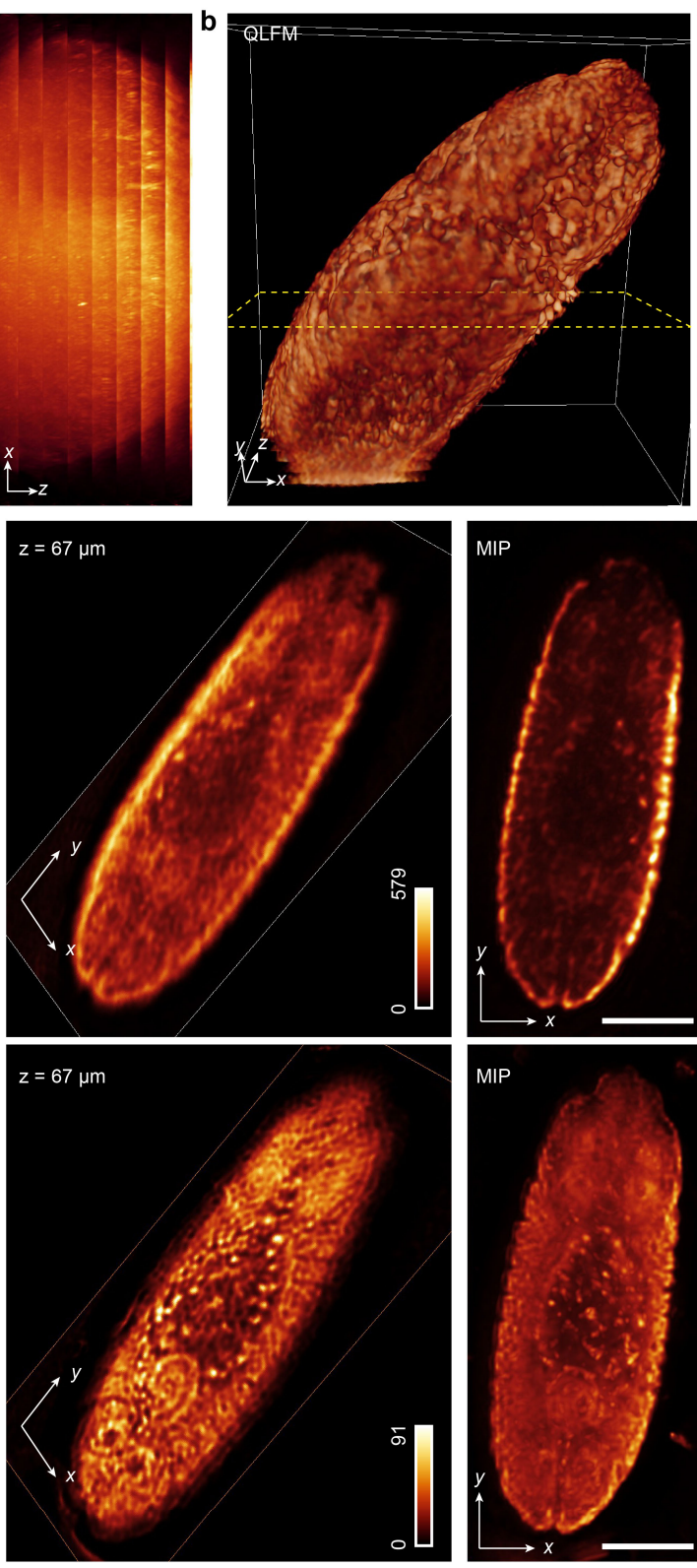
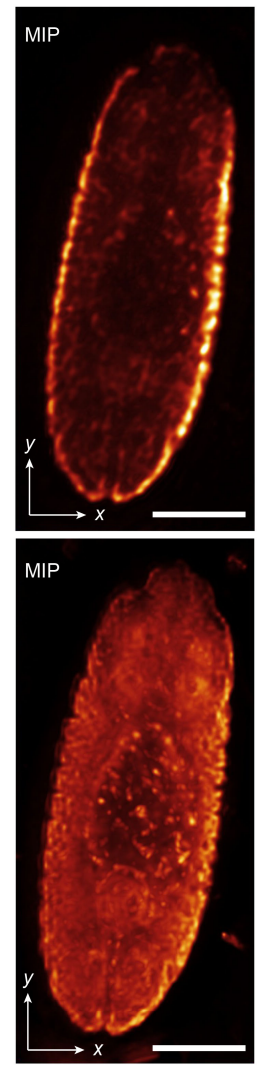
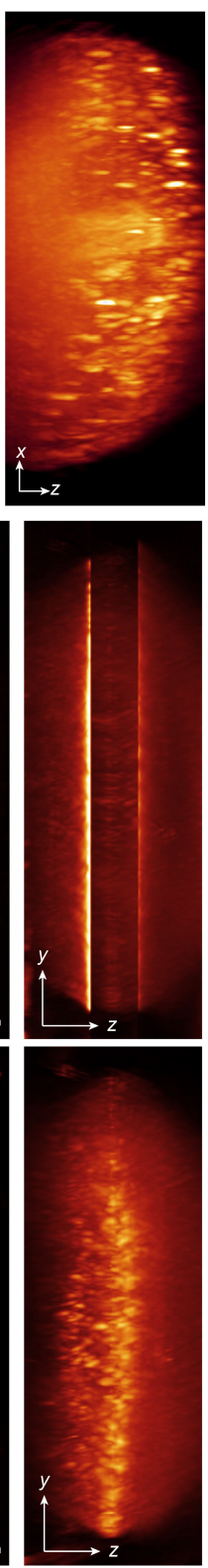

628

629

630

631

632

633

634

635

636

637

\section{Fig. 3 | Experimental comparisons on the Drosophila embryo with high-speed axial}

scanning. a-b, 3D rendered volumes and 180- $\mu \mathrm{m}-\mathrm{xz}$ MIPs at the same time point of traditional LFM and QLFM imaged with a 40×/1.0NA water-immersion objective at a step of $15 \mu \mathrm{m}$. QLFM show much better contrast and resolution than traditional LFM without artifacts, illustrating the capability of computational optical sectioning by QLFM with the improved penetration depth. c-d, The comparisons between traditional LFM and QLFM with a $20 \times / 0.5 \mathrm{NA}$ objective in the form of $3 \mathrm{D}$ rendered volumes, slices, and orthogonal MIPs with axial scanning at a step of $50 \mu \mathrm{m}$ indicating the uniform resolution of QLFM without edge artefacts. Scale bars, $50 \mu \mathrm{m}$ (a-b) and $100 \mu \mathrm{m}$ (c-d). 

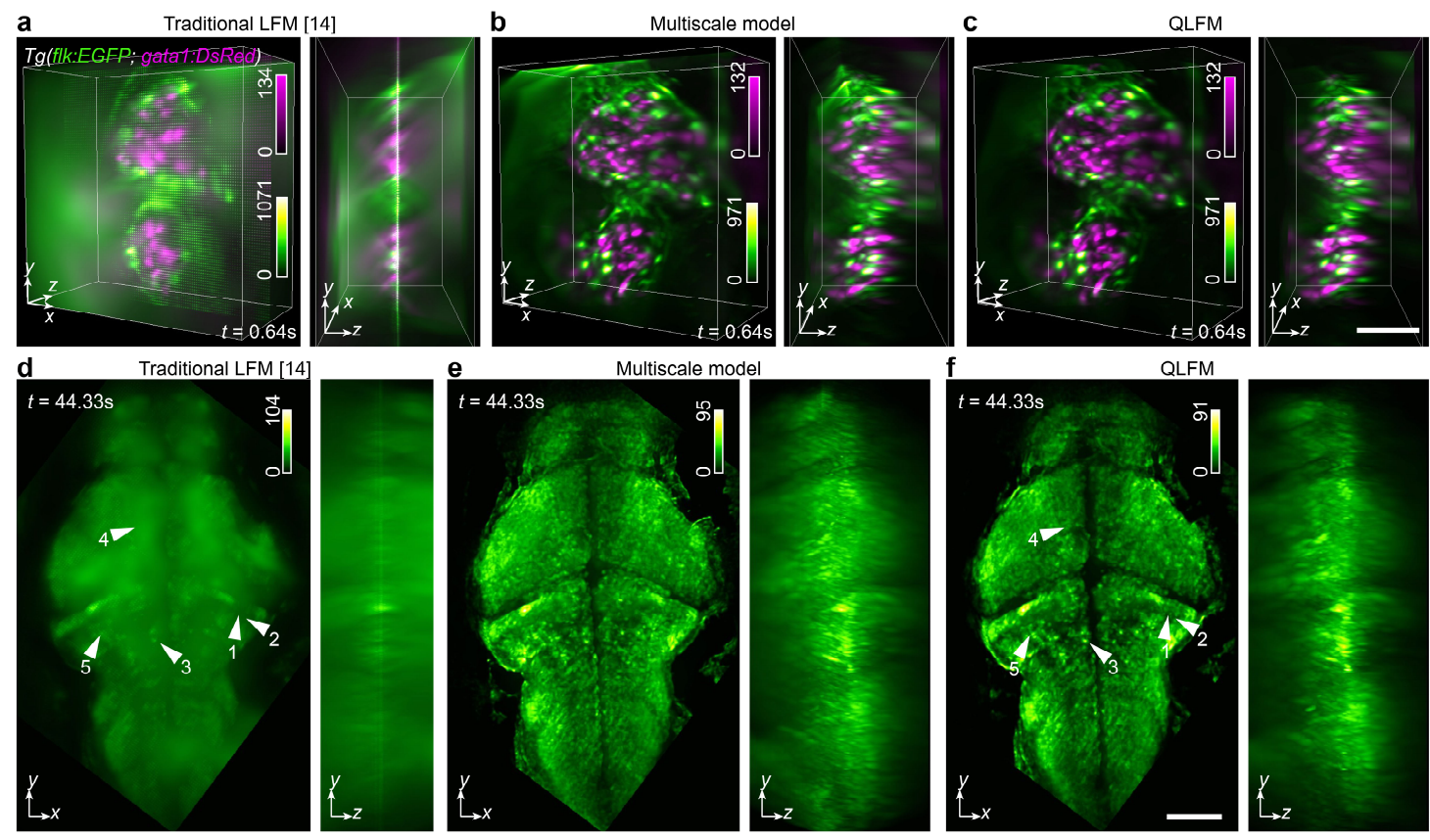

g
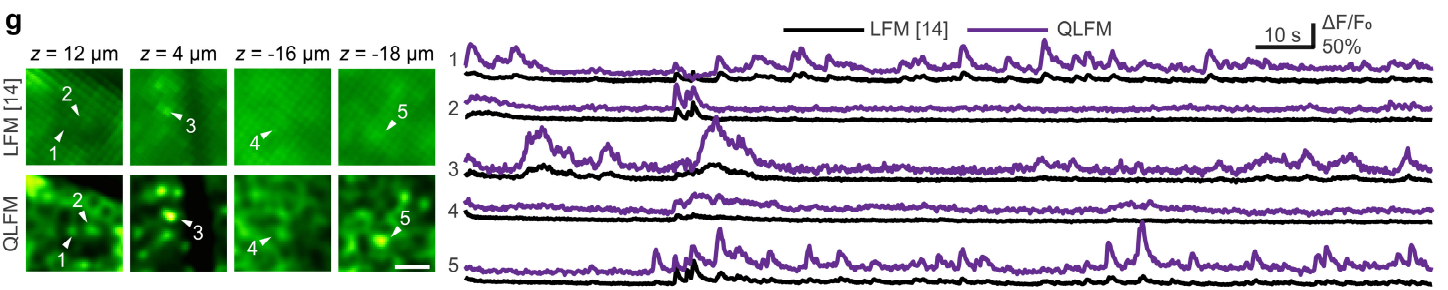

639 Fig. 4 | High-speed volumetric imaging in larval zebrafish. a-c, 3D rendered volumes

640 of the beating heart at the same time point reconstructed by the traditional LFM, QLFM

641 with the multiscale model only, and QLFM with the multiscale scattering model

642 (Supplementary Video 2). Successive improvements can be observed with significantly

643 reduced artifacts and background for quantitative imaging. d-f, Orthogonal maximum-

644 intensity projections (MIP) of the whole brain at the same time point reconstructed by the

645 traditional LFM, QLFM with the multiscale model only, and QLFM with the multiscale

646 scattering model, illustrating the effectiveness of computational optical sectioning in

647 densely labeled samples (Supplementary Video 3). g, Zoom-in slices of the same neurons

648 marked in $\mathrm{d}$ and $\mathrm{f}$ and their temporal traces for comparison, illustrating the improved

649 SBR. Scale bars, $50 \mu \mathrm{m}(\mathbf{a}-\mathbf{c}), 100 \mu \mathrm{m}(\mathbf{d}-\mathbf{f})$ and $20 \mu \mathrm{m}(\mathbf{g})$. 

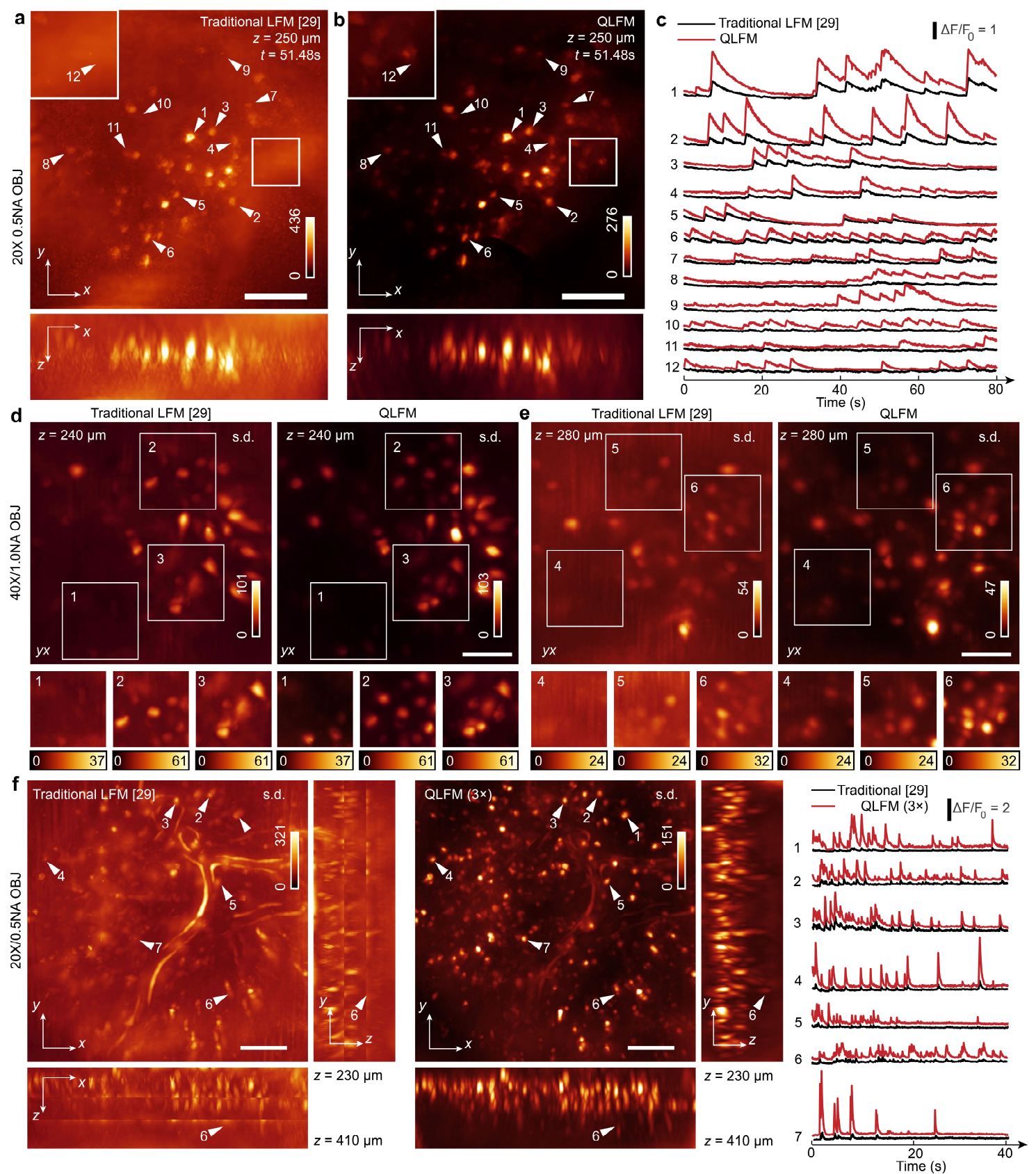

652 Fig. 5 | 3D functional imaging in awake mouse brains. a-b, Orthogonal MIPs of

653 GCaMP6s-labeled neurons at $t=51.48 \mathrm{~s}$ reconstructed by traditional LFM and QLFM with

654 a $20 \times / 0.5 \mathrm{NA}$ objective, indicating the reduced background with computational optical

655 sectioning (Supplementary Video 4). More neurons were revealed by QLFM in the mouse

656 cortex with the native objective plane at a depth of $\sim 250 \mu \mathrm{m}$. c, Temporal traces of the

657 marked neurons in a and $b$, demonstrating the quantitative calcium responses in QLFM

658 with improved contrast. d-e, Orthogonal MIPs of the standard deviation across 2000 
659 volumes imaged at a center depth of $240 \mu \mathrm{m}$ and $280 \mu \mathrm{m}$ in the cortex, respectively, under 660 a 40×/1.0 NA water-immersion objective (Supplementary Video 5). f, Orthogonal MIPs 661 of the standard deviation across 500 volumes for GCaMP6f-labeled L2/3 neurons in an

662 awake behaving mice. The video was captured with high-speed axial scanning at a step 663 of $50 \mu \mathrm{m}$ for 3 planes at $24 \mathrm{~Hz}$ with a $20 \times / 0.5 \mathrm{NA}$ objective. Temporal traces of several 664 marked neurons at different depths are shown on the right. QLFM shows uniform 665 resolution and consistent calcium responses across a large depth range, while there is 666 barely contrast in traditional LFM due to severe background. Scale bars, $100 \mu \mathrm{m}(\mathbf{a}-\mathbf{b}, \mathbf{f})$ 667 and $50 \mu \mathrm{m}(\mathbf{d}-\mathbf{e})$. 